\title{
Estrogen Enhances Endometrial Cancer Cells Proliferation by Upregulation of Prohibitin
}

\author{
Bin Yang ${ }^{1,2^{*}}$, Ruiying Chen ${ }^{3 *}$, Xiaoyan Liang1, Jiayan Shi ${ }^{1}$, Xiaomei $\mathrm{Wu}^{4,5}$, Zhenbo Zhang ${ }^{1,2,4,5}$,Xiong \\ Chen ${ }^{1,2}$ \\ 1. Department of Obstetrics and Gynecology, Shanghai First People's Hospital, Baoshan Branch, Shanghai, 201900, China \\ 2. Jiangxi Medical College, Nanchang University, Nanchang, 330000, China \\ 3. Department of Cervical Diseases, Shanghai First Maternity and Infant Hospital, Tongji University School of Medicine, Shanghai, 200040, China \\ 4. Reproductive Medicine, Department of Obstetrics and Gynecology,Shanghai First people's Hospital, Shanghai Jiaotong University, Shanghai, 201600,China \\ 5. Department of Obstetrics and Gynecology, Shanghai General Hospital, Shanghai Jiaotong University, Shanghai, 201600, China \\ *Contributed equally \\ $\triangle$ Corresponding author: Xiong Chen, Department of Obstetrics and Gynecology, Shanghai First People's Hospital, Baoshan Branch, Shanghai, 201900, \\ China.Tel:86-21-37798941;Fax:86-21-63240090. E-mail: smmuchenxiong@msn.com \\ (C) Ivyspring International Publisher. This is an open access article distributed under the terms of the Creative Commons Attribution (CC BY-NC) license \\ (https://creativecommons.org/licenses/by-nc/4.0/). See http://ivyspring.com/terms for full terms and conditions.
}

Received: 2018.06.30; Accepted: 2019.01.13; Published: 2019.03 .03

\begin{abstract}
Estrogen plays an essential role in type I endometrial cancer cell proliferation. Despite great progresses in the etiology has been obtained in the past, however, the molecular mechanisms remain to be fully clarified. Prohibitin has been demonstrated involvement in multiple cancers' development. If it also contributes to estrogen-driven endometrial cancer proliferation is not clear. IHC assay result display that prohibitin overexpressed in endometrial cancer tissue and associated with the poor prognosis; Western blot assay detect that upregulated prohibitin expression with dose- and time-dependent manners. The cellular growth was monitored with SRB assay which demonstrate that knockdown prohibitin attenuated estrogen-induced proliferation. Ubiquitination assay finds estrogen increased prohibitin level through stabilizing prohibitin protein via inhibition of ubiquitination, while estrogen-induced protein expression was mediated by estrogen receptor. Our findings provide a new insight on the mechanism of estrogen-induced proliferation, implying the possibility of using prohibitin as a potential therapeutic target for the treatment of endometrial cancer.
\end{abstract}

Key words: prohibitin, type 1 endometrial cancer, proliferation, estrogen, estrogen receptor

\section{Introduction}

Endometrial cancer is the most common gynecological malignancy worldwide and represents the eighth leading cause of cancer related death in women. Type 1 endometrial carcinoma are characterized by positive nuclear estrogen receptor (ER) expression and responsiveness to hormone stimulation. Increasing evidence indicates that prolonged estrogen exposure is associated with initiation of type 1 endometrioid cancers. Estrogen exposure results in an overall physiological response within several hours by a genomic mechanism which depends on estrogen binding to nuclear ER resulting in mRNA transcription and protein synthesis of target genes. Increasing evidence demonstrate that estrogen plays an essential role in type 1 endometrial occurrence, it was found that Protein Kinase C alpha [1], Malate dehydrogenase 2 (MDH2) [2], and $\mathrm{Ca}(2+)$ channel subunit alpha 1D [3], et al involve in endometrial cancer uncontrol proliferation. Consistent with others' study, we have stated that estrogen up-regulates FTO expression via activation of the PI3K/AKT and MPAK signal pathways, then further enhances endometrial cancer cell proliferation [4]. Moreover, it was found that notch signal pathway also contributes to estrogen-driven endometrial cancer cellular growth [5]. Although the great progress has been made in understanding how estrogen induces type 1 endometrial cancer, the detailed mechanism has not been revealed.

Aberrant prohibitin expression has been found in various cancer types and associates with tumorigenesis, such as breast, ovarian cancer, and gallbladder 
cancer [6-11]. Prohibitin has multiple roles in regulating cell biofunctions, including proliferation, mitochondrial respiration activity and aging [12]. Previously, it commonly believes that prohibitin plays an important role to suppress cancer development via inhibiting DNA synthesis [13]. Inversely, a mass of studies verify that prohibitin serve as an oncogene. Overexpression of prohibitin associates with poor prognosis in gallbladder cancer and breast cancer, moreover, it also promotes cancer proliferation and invasion. However, rare information can be available about the role of prohibitin in endometrial cancer development, especially in entrogen-driven proliferation. He B et. al has demonstrated that prohibitin is an estrogen-regulated gene in vitro and vivo, and its expression is induced by estrogen in the uterus [14]. Similar result has been observed in white adipose tissue and liver, and the estrogen-elevated prohibitin may mediated by estrogen receptor [15]. These results suggest that prohibtin involves in estrogen signaling.

Prohibitin ubiquitination has been found in mammalian sperm mitochondria, this posttranslational modification may regulate mitochondrial inheritance and sperm quality control [16]. More recent study found that estrogen could enhance estrogen receptor alpha ubiquitination and regulate breast cancer cell proliferation [17]. While, Minji Choi et. al pointed out that estrogen receptor mediates estrogen-induced prohitin expression. Therefore, if elevated prohibitin expression by estrogen associates deubiquitination signal pathway is not clear. Thus, the aim of this study is to investigate the expression pattern of prohibitin in endometrial cancer and explore how estrogen regulates endometrial cancer cell proliferation via estrogen signal pathway and whether involves in ubiquitination process.

\section{Materials and Methods}

\section{Cell lines and cell culture}

Endometrial cancer Ishikawa cell line was kept in our lab. It is an estrogen-responsive cell line derived from a well-differentiated endometrioid carcinoma. The cells were maintained in Dulbecco's modified Eagle's medium (DMEM) F-12 1:1 medium (GIBCO) with $10 \%$ fetal bovine serum (FBS; Gibco, Gaithersburg, MD, USA), $100 \mathrm{U} / \mathrm{ml}$ penicillin, sodium pyruvate and L-glutamine in a humidified atmosphere of $5 \% \mathrm{CO} 2$ at $37^{\circ} \mathrm{C}$.

\section{Plasmids, siRNA and transfection}

The pcDNA-ERa plasmid was kindly provided by Dr. Xiaoyue Chen (Department of Obstetrics and Gynecology, Shanghai First Maternity and Infant Hospital, Tongii University School of Medicine). siPHB was purchased from Shanghai GenePharma
Co., Ltd. The pcDNA-ERa plasmid was transfected in to ishikawa cells in the presence of Lipofectamine ${ }^{\mathrm{TM}}$ 3000 transfection reagent (Invitrogen, Carlsbad, CA, USA). Whereas prohibitin small interfering RNA was transfected in to ishikawa cells with hiperfect reagent. The siControl siRNA served as a transfection control. The overexpressing effect of pcDNA-ERa or knockdown efficiency of the siRNA were examined by immnuoblotting.

\section{Endometrial cancer specimens and IHC assay}

68 cases were enrolled in this study, which comprised 32 benign endometrial tissues and 36 cases of endometrial carcinoma. All the specimens were obtained from the Department of Obstetrics and Gynecology of the Shanghai General Hospital affiliated to Shanghai Jiao Tong University. None of patients in the study had a history of prior radiotherapy or chemotherapy. The use of these specimens was approved by the Ethics Committee of the Medical College, Shanghai Jiao Tong University, China. Pathological diagnoses of endometrial samples were made by two specialized gynecological pathologists based on the World Health Organization (WHO) classification for endometrial cancer in a double-blinded manner. IHC staining was performed as previous described. The sections were incubated with primary antibody against prohibitin (diluted to 1:100; Sigma, St. Louis, MO, USA ). The IHC scores were evaluated as previous described [4].

\section{Quantitative real time PCR}

Total RNA were extracted and reverse transcribed as previously described [4]. Primers for prohibitin and GAPDH genes were synthesized by Invitrogen Bioengineering Corporation (Shanghai, China) (Table 1). The reactions of real time PCR were set up with $10 \mu \mathrm{l}$ SYBR Green PCR Master Mix (Takara, Shuzo, Kyoto, Japan), $1.0 \mu 110 \mu \mathrm{M}$ primer mixture, and $2 \mu \mathrm{l}$ cDNA template. The PCR conditions were $95^{\circ} \mathrm{C}$ for $5 \mathrm{~min}$, followed by 35 cycles of $94^{\circ} \mathrm{C}$ for $1 \mathrm{~min}, 61^{\circ} \mathrm{C}$ for $30 \mathrm{~s}$ and $72^{\circ} \mathrm{C}$ for $45 \mathrm{~s}$. GAPDH serves as a control to normalize the expression of prohibitin gene. Each real time PCR experiment was repeated three times and a mean value was use for the determination of mRNA levels. The template replaced with water used as a negative control.

Table 1. Sequences of primers used for amplification of target genes

\begin{tabular}{ll}
\hline Gene & primer nucleotide sequence \\
\hline PHB & Forward: 5'-GTGCGCGAATCTGTGTTCAC -3' \\
& Reverse: 5'-GATAATGGGGTACTGGAACCAAG -3' \\
GAPDH & $\begin{array}{l}\text { Forward: 5'- AACGGATTTGGTCGTATTG-3' } \\
\text { Reverse: 5'- GGAAGATGGTGATGGGATT -3' }\end{array}$ \\
\hline
\end{tabular}




\section{SRB cell proliferation assay}

Ishikawa cells were seeded into 96-well plates at a concentration of $10 \times 10^{4} / \mathrm{ml}$. After overnight starvation in Opti-MEM medium, various treatments have been performed, and the cells were incubated another $48 \mathrm{hrs}$. The plates were then routinely processed with SRB staining as previously described $[18,19]$.

\section{Western blot analysis}

Western blotting was performed as previously reported $[4,19]$. Briefly, ishikawa cells with indicated treatments were harvested and lysised with RAPA buffer, after determination of the protein concentration, proteins $(50 \mu \mathrm{g})$ was loaded and separated by SDS-PAGE. After transferring to PVDF membranes, proteins were detected using specific antibodies. Each experiment was repeated at least three times.

\section{In vitro ubiquitination analysis}

Ishikawa cells were treated with DMSO or estrogen, respectively. The cells were harvested, lysed and boiled in a buffer containing 2\% SDS, $150 \mathrm{mM}$ $\mathrm{NaCl}, 10 \mathrm{mM}$ Tris- $\mathrm{HCl}$, and $1 \mathrm{mM}$ DTT. The lysates incubated with beads and probihitin antibody overnight at $4^{\circ} \mathrm{C}$. Immunoprecipitated proteins were analyzed by Western blotting using an anti-Ub antibody.

\section{Statistical analysis}

Comparisons among multiple groups were made with one-way analysis of variance (ANOVA) followed by Dunnet t-test. Statistical significance between the treated and untreated groups was

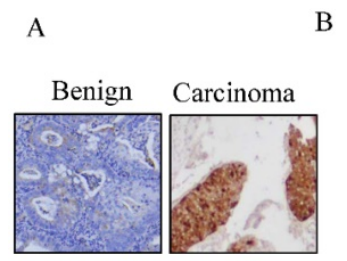

D

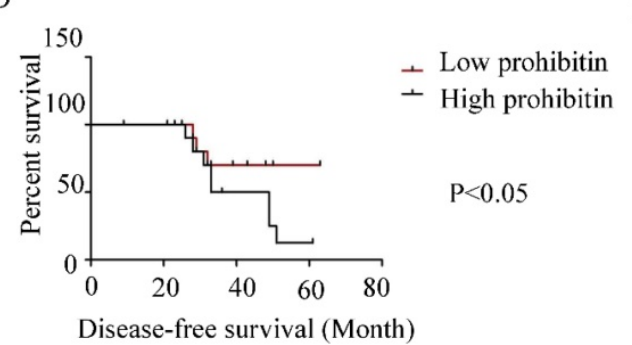

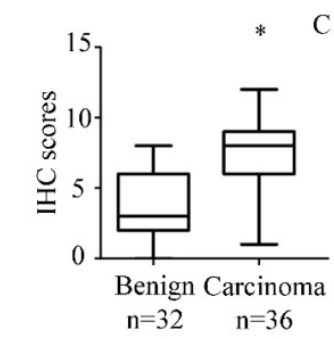
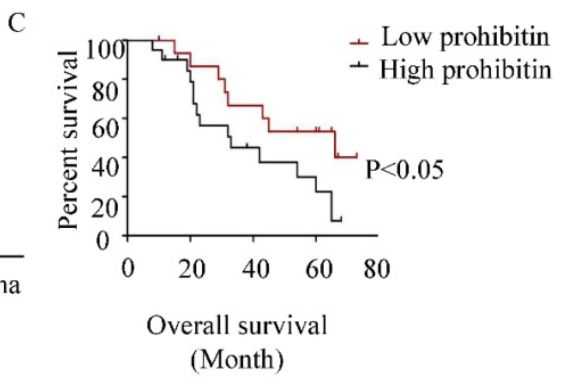

E

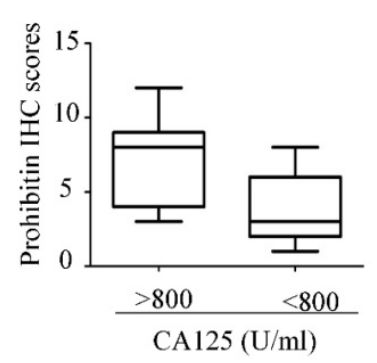

Figure 1. Prohibitin overexpresses in endometrial cancer and associates with poor prognosis. The expression of prohibitin in benign and endometrial cancer tissues was assessed using IHC staining. (A) Representative images were captured at $200 \times$ magnification. (B) Prohibitin IHC scores in endometrial lesion tissues are presented. (C, D) The overall survival and disease-free survival curves of endometrial cancer patients with different levels of prohibitin expression in 24 tissue samples of endometrial cancer. (E) Prohibitin expression correlated with serum CA125 level. *, $\mathrm{p}<0.05$. analyzed by Student's $\mathrm{t}$ test, and the statistical significance was set at $\mathrm{P}<0.05$.

\section{Results}

\section{Prohibitin overexpresses in endometrial cancer and associates with poor prognosis}

As shown in Figure 1 A\&B, higher expression of prohibitin was observed in endometrial carcinoma tissue than that in benign endometrial tissue. The percentage of positive staining in endometrial tissues and the immunohistochemistry scores for prohibitin staining are summarized in Figure $1 \mathrm{~B}$ and Table 2. The high expression of prohibitin was positively related to serum CA125 levels (Figure 1E). Moreover, high levels of prohibitin correlated with poor prognosis and early relapse (Figure $1 C \& D$ ).

Table 2. The expression of prohibitin in distinct tissue types

\begin{tabular}{llllll}
\hline Tissue type & Total & Positive & Negative & $\%$ & $P$-value \\
\hline Benign & 32 & 11 & 21 & 34.4 & 0.005 \\
Carcinomas & 36 & 27 & 9 & 75 & \\
\hline
\end{tabular}

\section{Estrogen upregulates prohibitin expression and promotes endometrial cancer cell proliferation}

To explore the effect of estrogen on prohibitin expression, western blot was performed. It was found that prohibitin protein expression elevated with the increasing dose of estrogen, the max peak of prohibitin expression was observed when it was exposed to $10 \mathrm{nM}$ estrogen (Figure 2A \& C). Moreover, prohibitin expression pattern also showed a time-depend manner (Figure 2B\&D). After 1nM estrogen treatment for $48 \mathrm{hrs}$, it showed the optimum stimulating effect on prohibitin expression, approximate 3 folds increasing of protein level was obtained. Meanwhile, we found that estrogen promoted endometrial cancer cell proliferation with a dose manner (Figure 2E). However, knockdown prohibitin not only suppressed endometrial cancer cellular growth, but also attenuated estrogen-induced proliferation (Figure 2F). These data implies that prohibitin plays an important role in 
estrogen-driven endometrial cancer cell proliferation.

\section{Estrogen mediates ubiquitination of prohibitin}

To further determine how estrogen regulates prohibitin expression, the total RNA was extracted after DMSO or 1nM estrogen treatment for $16 \mathrm{hrs}$ and real time PCR was performed. No significant difference in mRNA level was observed between above treatments (Figure 3A). However, 1nM estrogen potently elevated prohibitin protein level compared with DMSO-treated group (Figure 3B). Prior to harvest the cells, $\mathrm{CHX}$ was used to treat the cells for indicated time. As showed in figure 3C, estrogen treatment could enhance the prohibitin protein stability. Further study found that estrogen treatment reduced the ubiquitination of prohibitin (Figure 3D).

\section{Estrogen receptor alpha enhances prohibitin expression}

Commonly, estrogen receptor alpha plays an important role in estrogen-driven bio-activity. Therefore, the effect of estrogen receptor alpha on prohibitin expression was determined by western blot. It was found that transfection of pcDNA-ERa sharply upregulated prohibitin expression. Which suggests that estrogen receptor alpha enhances prohibitin expression.

\section{Estrogen receptor alpha is necessary for estrogen enhancing endometrial cancer proliferation via prohibitin}

To investigate the role of ERa in estrogeninduced prohibitin expression, ICI, the ERa inhibitor, was used to block ERa signaling before estrogen treatment. The inhibitor of ERa has no effect on prohibitin expression, but it could attenuate estrogenelevated prohibitin protein level (Figure 5A\&B). Conversely, we overexpressed ERa in endometrial cancer cells before estrogen treatment. It was found that increasing ERa could enhance prohibitin expression, moreover, transfection of ERa plasmid plus estrogen treatment resulted in the most higher level of prohibitin protein (Figure 5C\&D). Further study found that the expression profile of prohibitin parallized with the proliferative activity of endometrial cancer (Figure 5E). Estrogen treatment plus ERa transfection showed the most higher of cellular growth activity. These data suggest that ERa is necessary for estrogen-driven endometrial cancer cell proliferation via prohibitin.

\section{Discussion}

Type 1 endometrial cancer has clear precancerous stages, from disordered proliferative endometrium, hyperplasia, atypical hyperplasia, and progresses onward to endometrial cancer development and progression. Abundant studies revealed that estrogen plays an essential role in type 1 endometrial cancer cell proliferation as a high risk factor [20-22]. Numerous candidate molecules and signal pathways have been stated involvement in estrogen-driven endometrial oncogenesis [23-27]. To extend our understanding of endometrial cancer development, the estrogen signaling has been further studied.

The estrogen induced prohibitin expression is required for mouse uterine development and adult function [14]. It was demonstrated that selective ablation of the prohibitin allele in female mice resulted in severe uterus hypoplasty and sterile. This implies that estrogen signaling is pivotal for maintenance of normal female reproductive function. However, what will happen if the women underwent long-term unopposed estrogen exposure. We explored this question in the present study, estrogen treatment leaded to increase expression of prohibitin with doseand time-dependent manners in endometrial cancer cells. Moreover, estrogen facilitated endometrial cancer cell proliferation with a dose manner. Nevertheless, this enhanced effect was blocked by knockdown of prohibitin gene. Our results agree with above study [14] that estrogen signaling and its functions are mediated by prohibitin. That is to say prohibtin is also a candidate gene of estrogen signaling. Therefore, we further

Figure 2. Estrogen upregulates prohibitin expression and promotes endometrial cancer cell proliferation. $(\mathbf{A}, \mathbf{C})$ Estrogen upregulates prohibitin expression with a dose-dependent manner. (B, D) Estrogen upregulates prohibitin expression with a time cause. (E) Estrogen enhances endometrial cancer cell proliferation. (F) knockdown prohibitin attenuates estrogen-enhanced endometrial cancer cell proliferation. *, $\mathrm{p}<0.05$ compared with the control group. a, represents a significant difference compared with siCon; b, compared with siCon plus estrogen treatment. 
detected its expression profile in endometrial cancer clinical samples to identify the association between prohibitin and cancer occurrence. Overexpression of prohibitin was observed in endometrial carcinoma tissues compared with benign endometrial tissue, the endometrial cancer patients with high level of prohibitin showed lower survival rate. This suggests that prohibitin serves as a high risk factor for endometrial cancer development and a poor prognosis factor. Similar results also have been observed in gallbladder cancer and esophageal squamous cell carcinoma $[9,28]$. Interesting is that a positive association between prohibitin staining and CA125 serum level, a biomarker for diagnosis ovarian cancer and endometrial cancer, has been observed, which suggests that prohibitin plays an essential role in endometrial cancer development.

Estrogen receptors are important transcription factor to relay signals from estrogen and to regulate proliferation of some of hormone-related cancers. It is a key component of estrogen signal pathway. Previous study documented that prohibitin involves in estrogen receptor regulation via reprogramming of the SWI/SNF complex [29]. Conversely, it was found in current study that overexpression of estrogen receptor alpha significantly elevated prohibitin level, suggesting that prohibitin is regulated by estrogen receptor. ICI 182780 (ICI), a specific inhibitor for estrogen receptor, was used to block the estrogen receptor signaling. It was found that estrogeninduced prohibitin was attenuated by ICI treatment, estrogen stimulation could not rescue ICI induced inhibition effect. On the contrary, overexpression of estrogen receptor alpha enhanced estrogen-induced prohibitin expression. Above results suggest that estrogen receptor is required for transmitting estrogen stimulation signaling. Meanwhile, transfection of estrogen receptor alpha plasmid enhanced estrogeninduced endometrial cancer proliferative activity. The cellular growth effect is parallel with the expression profile of prohibitin after overexpression of estrogen receptor alpha plus estrogen treatment, which suggests that the estrogen/estrogen receptor signal pathway and its function were mediated by prohibitin.

In previous study, the ubiquitination of estrogen receptor has been well documented [30-33]. Rare study addressed its downstream proteins' stability. We found that estrogen could slightly upregulate prohibitin mRNA level and potently enhance protein level, whereas estrogen stimulation could postpone prohibitin protein degradation after CHX treatment. Further study demonstrated that estrogen treatment reduced the ubiquitination of prohibitin. These results imply that estrogen could stabilize the prohibitin protein by attenuating its degradation via ubiquitination signal pathway.
A
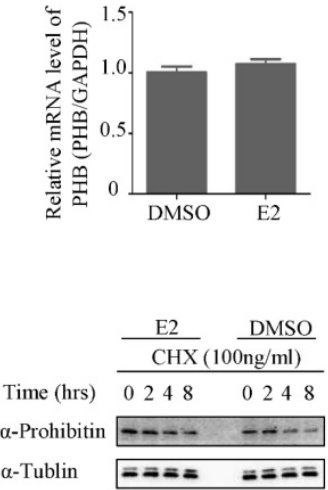

в
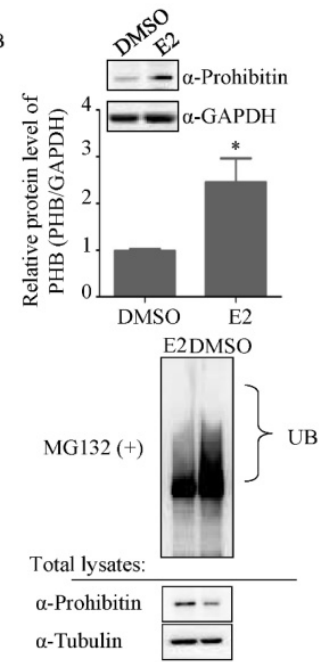

Figure 3. Estrogen mediates ubiquitination of prohibitin. (A) Estrogen ( $1 \mathrm{nM})$ slightly increases prohibitin mRNA level, which is determined by real time PCR, while the protein level is elevated by estrogen stimulation (B). (C) Estrogen enhances the stability of prohibitin protein. Ishikawa cells were treated with $100 \mathrm{ng} / \mathrm{ml}$ of cycloheximide ( $\mathrm{CHX}$ ) for the indicated times before $1 \mathrm{nM}$ estrogen treatment for $48 \mathrm{hrs}$. Prohitin protein levels were determined by western blot assay. (D) Estrogen treatment reduces the ubiquitination of prohibitin protein.

pcDNA3.0 +-
pcDNA-ERa -+
$\alpha$-Prohibitin -
$\alpha$-Tubulin

Figure 4. Estrogen receptor alpha enhances prohibitin expression. Western blot assay determines the expression of prohibitin after transfection of pcDNA-ERa plasmid (1 $\mu \mathrm{g})$.
A

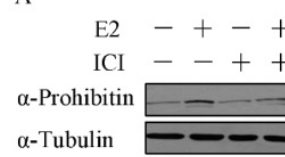

C

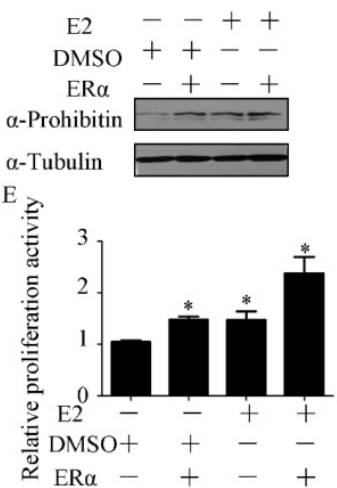

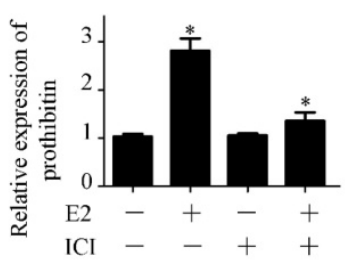

D

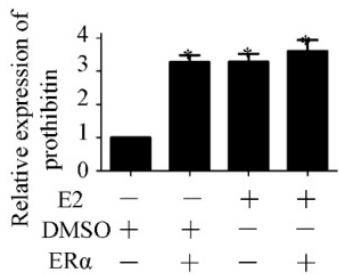

Figure 5. Estrogen receptor alpha is necessary for estrogen enhancing endometrial cancer proliferation via prohibitin. The effect of $\mathrm{ICl}$ on estrogen-induced prohibitin expression is determined by western blot $(\mathbf{A})$ and the statistical analysis of changes of prohibitin protein (B). $* P<0.05$, compared with control. Transfection pcDNA-ERa plasmid $(1 \mu \mathrm{g})$ enhances estrogen-induced prohibitin expression (C) and the statistical analysis of changes of prohibitin protein (D). $* \mathrm{P}<0.05$, compared with control. (E) Transfection of pCDNA-ERa plasmid $(1 \mu \mathrm{g})$ enhances estrogen-induced endometrial cancer proliferative activity. $* P<0.05$, compared with control. 
In summary, our findings revealed that estrogen promotes endometrial cancer cell proliferation by upregulation of prohibitin expression. Aberrant expression of prohibitin associates with poor endometrial cancer prognosis, which contributes to estrogendriven endometrial cancer development. Our findings highlighted the importance of prohibitin in estrogendriven endometrial cancer proliferation and suggest it as a potential prognostic marker and therapeutic target to improve the clinical management of endometrial cancer.

\section{Acknowledgements}

This study was funded by the National Natural Science Foundation of China [grant numbers 813022 55].

\section{Authors' contributions}

BY and RYC performed the experiments and wrote the manuscript; XYL, JYS, XMW contributed to the data analysis; $\mathrm{ZBZ}$ and $\mathrm{XC}$ revised the manuscript, and all authors have read and approved the final version of the manuscript.

\section{Competing Interests}

The authors have declared that no competing interest exists.

\section{References}

1. Thorne AM, Jackson TA, Willis VC, et al. Protein Kinase C alpha Modulates Estrogen-Receptor-Dependent Transcription and Proliferation in Endometrial Cancer Cells. Obstet Gynecol Int. 2013; 2013: 537479.

2. Zhuang $\mathrm{Y}$, Xiang $\mathrm{J}$, Bao $\mathrm{W}$, et al. MDH2 Stimulated by Estrogen-GPR30 Pathway Down-Regulated PTEN Expression Promoting the Proliferation and Invasion of Cells in Endometrial Cancer. Transl Oncol. 2017; 10: 203-10.

3. Hao J, Bao X, Jin B, et al. Ca2+ channel subunit alpha 1D promotes proliferation and migration of endometrial cancer cells mediated by 17beta-estradiol via the G protein-coupled estrogen receptor. FASEB J. 2015; 29: 2883-93.

4. Zhang Z, Zhou D, Lai Y, et al. Estrogen induces endometrial cancer cell proliferation and invasion by regulating the fat mass and obesity-associated gene via PI3K/AKT and MAPK signaling pathways. Cancer Lett. 2012; 319: 89-97.

5. Wei $\mathrm{Y}$, Zhang $\mathrm{Z}$, Liao $\mathrm{H}$, et al. Nuclear estrogen receptor-mediated Notch signaling and GPR30-mediated PI3K/AKT signaling in the regulation of endometrial cancer cell proliferation. Oncol Rep. 2012; 27: 504-10.

6. Zi Xu YX, Ande SR, Mishra S. Prohibitin: A new player in immunometabolism and in linking obesity and inflammation with cancer. Cancer Lett. 2018; 415: 208-16.

7. Liu $\mathrm{P}, \mathrm{Xu} \mathrm{Y}, \mathrm{Zhang} \mathrm{W}$, et al. Prohibitin promotes androgen receptor activation in ER-positive breast cancer. Cell Cycle. 2017; 16: 776-84.

8. El-Etreby NM, Ghazy AA, Rashad R. Prohibitin: targeting peptide coupled to ovarian cancer, luteinization and TGF-beta pathways. J Ovarian Res. 2017; 10: 28.

9. Cao $\mathrm{Y}$, Liang $\mathrm{H}$, Zhang $\mathrm{F}$, et al. Prohibitin overexpression predicts poor prognosis and promotes cell proliferation and invasion through ERK pathway activation in gallbladder cancer. J Exp Clin Cancer Res. 2016; 35: 68.

10. Webster LR, Provan PJ, Graham DJ, et al. Prohibitin expression is associated with high grade breast cancer but is not a driver of amplification at 17q21.33. Pathology. 2013; 45: 629-36.

11. Peng X, Mehta $R$, Wang $S$, et al. Prohibitin is a novel target gene of vitamin $D$ involved in its antiproliferative action in breast cancer cells. Cancer Res. 2006; 66: 7361-9.

12. Coates PJ, Nenutil R, McGregor A, et al. Mammalian prohibitin proteins respond to mitochondrial stress and decrease during cellular senescence. Exp Cell Res. 2001; 265: 262-73.

13. Fan W, Yang H, Liu T, et al. Prohibitin 1 suppresses liver cancer tumorigenesis in mice and human hepatocellular and cholangiocarcinoma cells. Hepatology. 2017; 65: 1249-66.
14. He B, Kim TH, Kommagani R, et al. Estrogen-regulated prohibitin is required for mouse uterine development and adult function. Endocrinology. 2011; 152: 1047-56.

15. Choi M, Chaudhari HN, Ji YR, et al. Effect of estrogen on expression of prohibitin in white adipose tissue and liver of diet-induced obese rats. Mol Cell Biochem. 2015; 407: 181-96.

16. Thompson WE, Ramalho-Santos J, Sutovsky P. Ubiquitination of prohibitin in mammalian sperm mitochondria: possible roles in the regulation of mitochondrial inheritance and sperm quality control. Biol Reprod. 2003; 69: 254-60.

17. Iizuka M, Susa T, Tamamori-Adachi M, et al. Intrinsic ubiquitin E3 ligase activity of histone acetyltransferase Hbo1 for estrogen receptor alpha. Proc Jpn Acad Ser B Phys Biol Sci. 2017; 93: 498-510.

18. Zou CF, Jia L, Jin H, et al. Re-expression of ARHI (DIRAS3) induces autophagy in breast cancer cells and enhances the inhibitory effect of paclitaxel. BMC Cancer. 2011; 11: 22

19. Tao X, Zhao N, Jin H, et al. FSH enhances the proliferation of ovarian cancer cells by activating transient receptor potential channel C3. Endocr Relat Cancer. 2013; 20: 415-29.

20. Chlebowski RT, Anderson GL, Sarto GE, et al. Continuous Combined Estrogen Plus Progestin and Endometrial Cancer: The Women's Health Initiative Randomized Trial. J Natl Cancer Inst. 2016; 108.

21. Dallal CM, Lacey JV, Jr., Pfeiffer RM, et al. Estrogen Metabolism and Risk of Postmenopausal Endometrial and Ovarian Cancer: the B approximately FIT Cohort. Horm Cancer. 2016; 7: 49-64.

22. Rozenberg S, Vasquez JB. Estrogen replacement therapy in patients with endometrial cancer: prescription attitude of Belgian gynecologists. Maturitas. 2000; 35: $125-8$.

23. Fujimoto J, Hori M, Ichigo S, et al. Estrogen induces expression of c-fos and c-jun via activation of protein kinase $C$ in an endometrial cancer cell line and fibroblasts derived from human uterine endometrium. Gynecol Endocrinol. 1996; 10: 109-18.

24. Skolnick AA. Estrogen trial for patients with endometrial cancer. JAMA. 1997; 278: 104 .

25. Sulak PJ. Endometrial cancer and hormone replacement therapy. Appropriate use of progestins to oppose endogenous and exogenous estrogen. Endocrinol Metab Clin North Am. 1997; 26: 399-412.

26. Chan S. A review of selective estrogen receptor modulators in the treatment of breast and endometrial cancer. Semin Oncol. 2002; 29: 129-33.

27. Acconcia F, Barnes CJ, Kumar R. Estrogen and tamoxifen induce cytoskeletal remodeling and migration in endometrial cancer cells. Endocrinology. 2006; 147: 1203-12.

28. Ren HZ, Wang JS, Wang $\mathrm{P}$, et al. Increased expression of prohibitin and its relationship with poor prognosis in esophageal squamous cell carcinoma. Pathol Oncol Res. 2010; 16: 515-22.

29. Zhang B, Chambers KJ, Faller DV, et al. Reprogramming of the SWI/SNF complex for co-activation or co-repression in prohibitin-mediated estrogen receptor regulation. Oncogene. 2007; 26: 7153-7.

30. Nirmala PB, Thampan RV. Ubiquitination of the rat uterine estrogen receptor: dependence on estradiol. Biochem Biophys Res Commun. 1995; 213: 24-31.

31. Ma Y, Fan S, Hu C, et al. BRCA1 regulates acetylation and ubiquitination of estrogen receptor-alpha. Mol Endocrinol. 2010; 24: 76-90.

32. Suzuki T, Fujii A, Ochi H, et al. Ubiquitination and downregulation of ErbB2 and estrogen receptor-alpha by kinase inhibitor MP-412 in human breast cancer cells. J Cell Biochem. 2011; 112: 2279-86.

33. Iizuka M, Susa $\mathrm{T}$, Takahashi $\mathrm{Y}$, et al. Histone acetyltransferase $\mathrm{Hbo1}$ destabilizes estrogen receptor alpha by ubiquitination and modulates proliferation of breast cancers. Cancer Sci. 2013; 104: 1647-55. 